\title{
P3: Evaluation des Projekts „Schüler leiten eine Station“ an einem Krankenhaus der Schwerpunktversorgung
}

\author{
Thomas Pfennig
}

Online publiziert: 23. Oktober 2013

(C) Springer-Verlag Wien 2013

Einleitung: Krankenpflegeschülerprojekte ermöglichen während der Ausbildung die geforderten theoretischen Lerninhalte mit den praktischen Übungen zu verknüpfen. Um die Sichtweise von Gesundheits- und Krankenpflegeschülern über die Vorbereitung und Durchführung eines selbstgestalteten 14-tägigen Schülerprojektes zu ermitteln, sollten diese Schüler nach einem solchen Projekt befragt werden.

Fragestellung: Am Beispiel des Projekts einer Schülerstation im Rahmen der Ausbildung von Gesundheits- und Krankenpflegeschülern an einem Krankenhaus der Schwerpunktversorgung soll untersucht werden, welche Ziele aus Sicht der Schüler realisiert werden konnten:

1. Haben die Schüler die Projektvorbereitung unterstützend für den Projektablauf empfunden?

2. Sind die Schüler mit dem Ablauf des Projektes zufrieden?

3. Wurden die zu Projektbeginn formulierten Ziele durch die Schüler realisiert?

4. Ist ein solches Projekt als optimale Prüfungsvorbereitung aus Sicht der Schüler zu sehen?

5. Welcher Zeitrahmen sollte in Zukunft für ein solches Projekt geplant werden?
Methodik: Alle 20 Gesundheits- und Krankenpflegeschüler im dritten Ausbildungsjahr eines Schülerprojekts erhielten einen anonymen Fragebogen. 18 Fragebögen wurden zurückgegeben.

Ergebnisse: Im Allgemeinen waren alle Schüler mit dem Ablauf des Projektes zufrieden, dieses wurde als eine sehr gute Vorbereitungsmöglichkeit auf die komplexe Abschlussprüfung gesehen. Das Projekt zeigte den Schülern sehr gut die Aufgaben eines Gesundheits- und Krankenpflegers. Die meisten Schüler gaben an, dass der gewählte Zeitrahmen zu kurz ist.

Diskussion/Schlussfolgerungen: Das Schülerprojekt wurde insgesamt gut angenommen und als Bereicherung der Ausbildung angesehen. Perspektivisch sollte jedoch geklärt werden, welche Zeitdauer für ein Schülerprojekt anzustreben ist.

T. Pfennig $(\bowtie)$

Studiengang Pflegemanagement, Studienzentrum Dresden,

Hamburger Fernhochschule, Hamburg, Deutschland

E-Mail: tpfennig.qft@googlemail.com 